\title{
Evaluating the Effect of Different Sources of Zinc on Egg Production, Egg Quality, and Hatchability Traits in Chickens
}

\author{
Siddhartha Shankar Pathak ${ }^{1 *}$, Prasoon Sagunan², \\ Biju Borah $^{1}$ and Prasanta Chabudhara ${ }^{1}$
}

\author{
${ }^{1}$ Lakhimpur College of Veterinary Science, Joyhing, North Lakhimpur, Assam, India \\ ${ }^{2}$ College of Avian Sciences and Management, Thiruvazhamkunnu, Kerala, India
}

*Corresponding author

\section{A B S T R A C T}

Keywords

Egg quality, Fertility,

Hatchability, Layer performance, Zinc sources

\section{Article Info}

\section{Accepted:}

07 January 2021

Available Online:

10 February 2021
Reports on feeding of zinc not only improved the health status of the chicken but also improved egg production, hatchability, and fertility and quality parameters. Different sources of zinc are available in the market for feeding. The current study aimed to assess the effect of dietary supplementation of inorganic, organic, and nano zinc on egg production, egg quality, and hatchability traits in white Plymouth Rock chickens. A total of 252 wings banded White Plymouth Rock birds of 33 weeks age were randomly assigned in seven groups. The diet was supplemented with inorganic, organic @ 30 and 60 mg/kg while nano zinc @ 15, 30 and $60 \mathrm{mg} / \mathrm{kg}$. Egg production, fertility, hatchability, egg quality parameters changes were recorded at different phases. It was suggestive that a higher level of nano zinc supplementation @ $60 \mathrm{mg} / \mathrm{kg}$ can be considered as a replacement of both inorganic and organic sources of zinc supplementation with better results.

\section{Introduction}

Zinc is a component of the uterine enzyme carbonic anhydrase, which supports eggshell formation (Nys et al., 2011). Zinc indirectly affects the quality of the epithelium due to its role in protein synthesis. The deficiency of Zinc in the diet decreases egg production and increases the chances of reproductive disease conditions. Zinc is a component of Carbonic anhydrase, which is an essential enzyme for eggshell formation. Three different sources of zinc are available for the feed industry viz inorganic, organic, and nano zinc. Due to the higher bioavailability of $\mathrm{Zn}$ from nano-zinc than inorganic and organic sources, they have better absorption than the other two sources resulting in better growth Pathak et al., (2020). Nano zinc (nZn) represents one of the nanotechnological approaches to prepare mineral salt having a particle size of 1 to 100nm Feng et al., (2009). Keeping these findings in mind, a study was framed to assess the effect of dietary supplementation of inorganic, organic, and nano zinc on egg production, egg quality, and hatchability traits in White Plymouth Rock chickens. 


\section{Materials and Methods}

A total of 252 wings banded White Plymouth Rock birds of 33 weeks age were randomly assigned to seven groups with four replicates in each group having 09 birds in each replicate (36 birds per treatment) in a deep litter system with all standard managemental practices were used for the study up to 53 weeks of age. The duration was divided into four phases viz. Phase I from $33^{\text {rd }}$ to $37^{\text {th }}$ week, Phase II $38^{\text {th }}$ to $42^{\text {nd }}$ week, Phase III $43^{\text {rd }}$ to $47^{\text {th }}$ week, Phase IV $48^{\text {th }}$ to $52^{\text {nd }}$ week. Birds were immunized as per the standard vaccination schedule and were fed with layer mash with one of the experimental diets. The experimental diets (mash form) were formulated as per BIS (2007) specifications. The ingredient composition, calculated nutrient values, and analyzed nutrient values of the basal (Table 1). Experimental feed samples were analyzed for the proximate composition according to the AOAC (2012) with a Zinc concentration of $30 \mathrm{ppm}$ by atomic absorption spectrophotometry (ICPOES; Perkin Elmer Optima 8000) in the basal diet. Dietary additions of $\mathrm{Zn}$ were made with $\mathrm{Zn}$ being provided with one of the following seven diets. To formulate $T_{1}$ and $T_{2}$, zinc sulphate at 30 and $60 \mathrm{mg} / \mathrm{kg}$ of diet respectively was added. To formulate $T_{3}$ and $\mathrm{T}_{4}$, zinc methionine at 30 and $60 \mathrm{mg} / \mathrm{kg}$ of diet respectively. For $\mathrm{T}_{5}, \mathrm{~T}_{6}$, and $\mathrm{T}_{7}$, nano zinc was added to the diet to contain $15 \mathrm{mg}, 30 \mathrm{mg}$, and $60 \mathrm{mg}$ levels of zinc respectively. Each bird received $125 \mathrm{~g} /$ day at the beginning of the trial and the feed allocation was gradually increased as the egg production increased. Based on the number of eggs produced in each phase, the hen housed egg production (HHEP) and hen day egg production (HDEP) were calculated. Eggs collected from the experimental hens were tested for their fertility as well as hatchability percent set during each period. Eggs collected during $37^{\text {th }}, 42^{\text {nd }}, 47^{\text {th }}$, and $52^{\text {nd }}$ week were analyzed for egg quality parameters. Two eggs from each replicate and eight eggs from each treatment group were taken for the study. The eggs collected for quality analysis were weighed, measured, and broken on the same day to assess the following external and internal quality parameters. The experiment was conducted upon approval of Institutional Ethics Approval Committee guidelines. Data on various parameters obtained during the trial were analyzed statistically by ANOVA using SPSS 20 statistical software. Differences between the means were tested using Duncan's Multiple Range Test Duncan (1995) at $\mathrm{P}<0.05$.

\section{Results and Discussion}

\section{Egg production}

During the phase, I, the HHEP percent, and HDEP percent significantly $(\mathrm{P} \leq 0.05)$ differed among different treatment groups. HHEP ranged from 71.34 percent in the $T_{1}$ group to 83.01 percent in $\mathrm{T}_{7}$ (Nano zinc, $60 \mathrm{mg} / \mathrm{kg}$ ). HHEP was significantly $(\mathrm{P} \leq 0.05)$ more in all groups when compared to $\mathrm{ZnSo}_{4}$ groups (Table 2). Among Zn-Met treatment groups, the difference was comparable, the HHEP in $\mathrm{T}_{4}$ (Zn-Met, $60 \mathrm{mg} / \mathrm{kg}$ ) was comparable to that of $\mathrm{T}_{5}$ (Nano zinc, $15 \mathrm{mg} / \mathrm{kg}$ ). The effect of different treatments on the HDEP percent was similar to that of HHEP. The HDEP ranged from 71.34 percent $\left(T_{1}\right)$ to 83.01 percent in the $\mathrm{T}_{7}$ group. A significantly $(\mathrm{P} \leq 0.05)$ higher percent of HDEP was recorded in all groups when compared to the $\mathrm{T}_{1}$ group. The differences in $\mathrm{Zn}-\mathrm{Met}$ treated groups were comparable to that of $T_{5}$ nano zinc supplemented group $T_{5}$ (nano zinc, 15 $\mathrm{m} / \mathrm{kg}$ ). Among $\mathrm{ZnSo}_{4}$ groups, the difference was insignificant and was comparable for HDEP percent. The results obtained are under the findings of (Klecker et al., 2002; Fakler et al., 2002) who reported an increase in egg production by supplementing organic zinc. 
These results are also similar to the reports of (Amen and Daraji 2012; Yang et al., 2012) reported an increase in egg production by supplementation of zinc. The findings have contradicted the reports of Guo et al., (2002) reported no effect on egg production in laying hens fed diets supplemented with inorganic zinc (zinc sulfate). The significant increase in egg production of the $T_{7}$ group might be due to the important role of $\mathrm{Zn}$ in the synthesis and secretion of $\mathrm{LH}$ and FSH hormones (Bedwal and Bahuguna, 1994). Dietary zinc may influence egg production by interacting with the endocrine system since the hen is changing the production and secretion of reproductive hormones during sexual maturation Renema et al., (1999).

During the rest of the phases (II-IV), HHEP and HDEP percent were significantly better in $\mathrm{T}_{7}$ (Nano zinc, $60 \mathrm{mg} / \mathrm{kg}$ ) when compared to $\mathrm{ZnSo}_{4}$ groups $\left(\mathrm{T}_{1}\right.$ and $\left.\mathrm{T}_{2}\right)$. But the difference was insignificant $(\mathrm{P} \leq 0.05)$ when compared to the rest of the treatment groups. Among $\mathrm{ZnSo}_{4}$ groups and $\mathrm{Zn}-\mathrm{Met}$ groups, the difference was insignificant $(\mathrm{P} \leq 0.05)$ respectively. The HHEP and HDEP percent in $\mathrm{T}_{1}$ was comparable to that of $\mathrm{T}_{2}$ and $\mathrm{T}_{3}$ with $\mathrm{T}_{4}$. Findings of Kout El-Kloub et al., (2004) are also in line with the present findings, displayed that supplementing the diet of Egyptian laying hens with zinc oxide or $\mathrm{Zn}$ methionine at two levels of 100 or 150 ppm resulted in significantly greater egg production and are comparable. Similar reports are presented by Hudson et al., (2004) in broiler breeders reported partial $(50 \%)$ or complete substitution of zinc sulphate with zinc amino acid complexes increased hen-day production, but only in the early phase of laying cycle (24 to 37 weeks of age). The findings of the present study are not in line with the findings of Drumus et al., (2004), who reported a non-significant effect of increasing the zinc level in the diet with different sources of zinc. The increase in egg production in zinc treatment groups' may be attributed to the increase in blood plasma sex hormones concentrations as was found a significant positive correlation among these hormones concentrations in blood plasma and egg production Durmus et al., (2004).

\section{Fertility}

During the I phase, the mean fertility percentage was non-significant between the treatment groups, however, a comparably higher percentage of fertility was recorded for the $\mathrm{T}_{7}$ group (Table 3). Similar findings are also reported by (Kidd et al., 1992), wherein a non-significant difference was recorded in hens fed with $\mathrm{ZnO}$ and $\mathrm{Zn}-$ Met.

During the rest of the three phases (II-IV) for fertility percentage, a significantly $(\mathrm{P} \leq 0.05)$ higher percent of fertility was recorded for the $\mathrm{T}_{7}$ group when compared between the treatment groups. However, the difference was comparable with the Zn-Met treatment group $\left(\mathrm{T}_{4}\right)$ and Nano zinc supplemented group at the levels of 15 and $30 \mathrm{mg} / \mathrm{kg}$. The reason for the comparable fertility percentage between Zn-Met at the level of $60 \mathrm{mg} / \mathrm{kg}$ with nano zinc at the level of $15 \mathrm{mg} / \mathrm{kg}$ might be attributed to similarity in bioavailability. The findings for increased fertility in the $\mathrm{T}_{7}$ group are also supported by the findings of Kout ElKoub et al., (2004) who reported the addition of a higher level of Zn-Met increased fertility percentage. The reason could be attributed to the higher bioavailability of nano zinc particles compared to other sources of zinc supplementation. Zinc is involved in the production, storage, and secretion of reproductive hormones and affects receptor sites (McDowell, 1992). Among $\mathrm{ZnSo}_{4}$ groups, the difference was comparable between them with the lowest value (87.05) recorded for the $\mathrm{T}_{1}$ group. Similar findings are also reported by Slamony et al., (2015), wherein an increase of fertility percent was 
recorded in $125 \mathrm{mg}$ than $100 \mathrm{mg} / \mathrm{kg}$ of Zn-Gly supplementation. The findings have contradicted the reports of (Kidd et al., 1992), wherein diet supplemented with $\mathrm{Zn}$-oxide and $\mathrm{Zn}$-Met did not differ the fertility percentage significantly.

\section{Hatchability}

A significantly $(\mathrm{P} \leq 0.05)$ higher hatchability on TES was recorded for the $\mathrm{T}_{7}$ group between the treatment groups which was persistent throughout different phases (Table 4). However, the difference was comparable to Zn-Met groups and other two nano zinc fed treatment groups. The difference for $\mathrm{ZnSo}_{4}$ groups was comparable to $\mathrm{Zn}-\mathrm{Met}$ groups and other two nano zinc fed treatment groups at the level of 15 and $30 \mathrm{mg} / \mathrm{kg}$. The $\mathrm{Zn}-\mathrm{Met}$ treatment groups were comparable to that of nano zinc supplemented groups. King'ori (2011) reported that among the most influential egg parameters that influence hatchability is weight, shape index and shell thickness. The reason might be attributed to higher egg weight of the $T_{7}$ treatment group was significantly higher than other treatment groups.

Table.1 Ingredient composition and nutrient composition of the basal diet

\begin{tabular}{|c|c|}
\hline Ingredients & Percentage \\
\hline Yellow maize & 63.35 \\
\hline Soyabean meal $(46 \%)$ & 23.00 \\
\hline DORB & 1.50 \\
\hline Vegetable oil & 1.50 \\
\hline Shell grit & 8.40 \\
\hline Dicalcium phosphate & 1.00 \\
\hline Mineral mixture * & 0.55 \\
\hline Vitamin premix ** & 0.10 \\
\hline DL-methionine & 0.10 \\
\hline Common salt & 0.40 \\
\hline TOTAL & 99.967 \\
\hline \multicolumn{2}{|l|}{ Nutrient Composition } \\
\hline ME (Kcal/kg) ${ }^{a}$ & 2834 \\
\hline Crude protein $(\%)^{b}$ & 16.5 \\
\hline Calcium $(\%)^{a}$ & 3.62 \\
\hline Phosphorous (\%) ${ }^{\text {a }}$ & 0.42 \\
\hline Lysine $(\%)^{\mathrm{a}}$ & 0.87 \\
\hline Methionine (\%) ${ }^{\mathrm{a}}$ & 0.47 \\
\hline
\end{tabular}

* Mineral mixture: Each $100 \mathrm{~g}$ contains Magnesium oxide- $1.48 \mathrm{~g}$, Ferrous sulphate- $6.0 \mathrm{~g}$, copper sulphate0.05g, Manganese Sulphate-0.04 g, Potassium Iodide- 0.001g, Potassium Chloride-17.09g and Sodium selenite- $0.001 \mathrm{~g}$.

** Vitamin-mineral Premix: Each 100g contains Vitamin AD3 (Vitamin A-10,00,000 IU/g, Vitamin D$200000 \mathrm{IU} / \mathrm{g}$ )- $0.165 \mathrm{~g}$, Vitamin K3-0.103g, Vitamin E- 2.4g, Thiamine Mononitrate- $0.206 \mathrm{~g}$, Riboflavin$0.513 \mathrm{~g}$, Pyridoxine hydrochloride- $0.309 \mathrm{~g}$, Cyanocobalamine- $0.00031 \mathrm{~g}$, Folic acid- $0.103 \mathrm{~g}$, Niacin- $4.124 \mathrm{~g}$, Ca-D-Pantothenate- $1.031 \mathrm{~g}$, Biotin- $1.5 \mathrm{~g}$, Maltodextrine- $89.545 \mathrm{~g}$.

${ }^{\mathrm{a}}$ calculated values; ${ }^{\mathrm{b}}$ analyzed values 
Table.2 Effect of supplementing ZnSo4, Zn-Met and nano zinc on egg production (\%) of White Plymouth Rock bird

\begin{tabular}{|c|c|c|c|c|c|c|c|c|c|c|}
\hline \multirow{2}{*}{$\begin{array}{l}\text { Treat- } \\
\text { ment }\end{array}$} & \multirow{2}{*}{$\begin{array}{c}\text { Zinc } \\
\text { source }\end{array}$} & \multirow{2}{*}{$\begin{array}{c}\text { Level } \\
\text { (mg/ } \\
\text { kg) }\end{array}$} & \multicolumn{2}{|c|}{ Phase I } & \multicolumn{2}{|c|}{ Phase II } & \multicolumn{2}{|c|}{ Phase III } & \multicolumn{2}{|c|}{ Phase IV } \\
\hline & & & $\begin{array}{c}\text { HHEP } \\
(\%)\end{array}$ & $\begin{array}{c}\text { HDEP } \\
(\%)\end{array}$ & $\begin{array}{c}\text { HHEP } \\
(\%)\end{array}$ & $\begin{array}{c}\text { HDEP } \\
(\%)\end{array}$ & $\begin{array}{c}\text { HHEP } \\
(\%)\end{array}$ & $\begin{array}{c}\text { HDEP } \\
(\%)\end{array}$ & $\begin{array}{c}\text { HHEP } \\
(\%)\end{array}$ & $\begin{array}{c}\text { HDEP } \\
(\%)\end{array}$ \\
\hline $\mathbf{T 1}$ & $\mathrm{ZnSo}_{4}$ & 30 & $\begin{array}{c}71.34 \pm \\
1.42^{\mathrm{a}}\end{array}$ & $\begin{array}{c}71.34 \pm \\
1.42^{\mathrm{a}}\end{array}$ & $\begin{array}{c}67.85 \pm \\
1.13^{\mathrm{a}}\end{array}$ & $\begin{array}{c}69.79 \pm \\
1.16^{\mathrm{a}}\end{array}$ & $\begin{array}{c}66.03 \pm \\
0.88^{\mathrm{a}}\end{array}$ & $\begin{array}{c}67.91 \pm \\
0.90^{\mathrm{a}}\end{array}$ & $\begin{array}{c}64.68 \pm \\
1.42^{\mathrm{a}}\end{array}$ & $\begin{array}{c}66.53 \pm \\
1.46^{\mathrm{a}}\end{array}$ \\
\hline $\mathbf{T} 2$ & $\mathrm{ZnSo}_{4}$ & 60 & $\begin{array}{c}73.49 \pm \\
1.13^{\mathrm{a}}\end{array}$ & $\begin{array}{c}73.49 \pm \\
1.13^{\mathrm{a}}\end{array}$ & $\begin{array}{c}70.79 \pm \\
1.27^{\mathrm{ab}}\end{array}$ & $\begin{array}{c}72.81 \pm \\
1.30^{\mathrm{ab}}\end{array}$ & $\begin{array}{c}67.77 \pm \\
0.89^{\mathrm{a}}\end{array}$ & $\begin{array}{c}69.71 \pm \\
0.92^{\mathrm{a}}\end{array}$ & $\begin{array}{c}66.03 \pm \\
1.10^{\mathrm{ab}}\end{array}$ & $\begin{array}{c}67.91 \pm \\
1.13^{\mathrm{ab}}\end{array}$ \\
\hline T3 & Zn-Met & 30 & $\begin{array}{c}75.00 \pm \\
1.13^{\mathrm{ab}}\end{array}$ & $\begin{array}{c}75.00 \pm \\
1.13^{\mathrm{ab}}\end{array}$ & $\begin{array}{c}72.53 \pm \\
2.07^{b}\end{array}$ & $\begin{array}{c}74.61 \pm \\
2.13^{b}\end{array}$ & $\begin{array}{c}69.36 \pm \\
1.24^{\mathrm{a}}\end{array}$ & $\begin{array}{c}71.34 \pm \\
1.28^{\mathrm{a}}\end{array}$ & $\begin{array}{c}68.73 \pm \\
1.07^{\mathrm{b}}\end{array}$ & $\begin{array}{c}68.73 \pm \\
1.07^{\mathrm{b}}\end{array}$ \\
\hline $\mathbf{T 4}$ & Zn-Met & 60 & $\begin{array}{c}77.38 \pm \\
1.46^{\mathrm{b}}\end{array}$ & $\begin{array}{c}77.38 \pm \\
1.46^{\mathrm{b}}\end{array}$ & $\begin{array}{l}74.44 \pm \\
1.45^{\mathrm{bc}}\end{array}$ & $\begin{array}{l}74.44 \pm \\
1.45^{\mathrm{bc}}\end{array}$ & $\begin{array}{c}73.49 \pm \\
1.15^{\mathrm{b}}\end{array}$ & $\begin{array}{c}75.59 \pm \\
1.19^{\mathrm{b}}\end{array}$ & $\begin{array}{c}72.53 \pm \\
1.43^{c}\end{array}$ & $\begin{array}{c}74.61 \pm \\
1.47^{\mathrm{c}}\end{array}$ \\
\hline T5 & NanoZinc & 15 & $\begin{array}{c}78.09 \pm \\
1.44^{\mathrm{bc}}\end{array}$ & $\begin{array}{c}78.09 \pm \\
1.44^{\mathrm{bc}}\end{array}$ & $\begin{array}{c}74.75 \pm \\
1.39^{\mathrm{bc}}\end{array}$ & $\begin{array}{c}74.75 \pm \\
1.39^{\mathrm{bc}}\end{array}$ & $\begin{array}{c}73.65 \pm \\
1.31^{b}\end{array}$ & $\begin{array}{c}75.75 \pm \\
1.34^{\mathrm{b}}\end{array}$ & $\begin{array}{c}72.38 \pm \\
1.37^{\mathrm{c}}\end{array}$ & $\begin{array}{c}74.44 \pm \\
1.41^{\mathrm{c}}\end{array}$ \\
\hline T6 & $\begin{array}{l}\text { Nano } \\
\text { Zinc }\end{array}$ & 30 & $\begin{array}{c}81.26 \pm \\
1.05^{\mathrm{cd}}\end{array}$ & $\begin{array}{c}81.26 \pm \\
1.05^{\mathrm{cd}}\end{array}$ & $\begin{array}{c}76.82 \pm \\
1.38^{c}\end{array}$ & $\begin{array}{c}76.82 \pm \\
1.38^{c}\end{array}$ & $\begin{array}{c}75.39 \pm \\
1.17^{\text {bc }}\end{array}$ & $\begin{array}{c}77.55 \pm \\
1.21^{\mathrm{bc}}\end{array}$ & $\begin{array}{c}74.20 \pm \\
1.12^{\mathrm{c}}\end{array}$ & $\begin{array}{c}76.32 \pm \\
1.15^{\mathrm{c}}\end{array}$ \\
\hline $\mathbf{T 7}$ & $\begin{array}{l}\text { Nano } \\
\text { Zinc }\end{array}$ & 60 & $\begin{array}{c}83.01 \pm \\
1.17^{\mathrm{d}}\end{array}$ & $\begin{array}{c}83.01 \pm \\
1.17^{\mathrm{d}}\end{array}$ & $\begin{array}{c}78.49 \pm \\
1.45^{\mathrm{c}}\end{array}$ & $\begin{array}{c}78.49 \pm \\
1.45^{\mathrm{c}}\end{array}$ & $\begin{array}{c}77.22 \pm \\
1.29^{c}\end{array}$ & $\begin{array}{c}79.42 \pm \\
1.33^{\mathrm{c}}\end{array}$ & $\begin{array}{c}73.65 \pm \\
1.32^{c}\end{array}$ & $\begin{array}{c}75.75 \pm \\
1.36^{\mathrm{c}}\end{array}$ \\
\hline
\end{tabular}

Means having same superscript do not differ significantly

Table.3 Effect of supplementing $\mathrm{ZnSo}_{4}, \mathrm{Zn}-\mathrm{Met}$ and nano zinc on fertility (\%) of White Plymouth Rock bird

\begin{tabular}{|c|c|c|c|c|c|c|}
\hline \multirow{2}{*}{$\begin{array}{l}\text { Treat- } \\
\text { ment }\end{array}$} & \multirow{2}{*}{$\begin{array}{c}\text { Zinc } \\
\text { source }\end{array}$} & \multirow{2}{*}{$\begin{array}{c}\text { Level } \\
\text { (mg/kg } \\
\text { ) }\end{array}$} & \multicolumn{4}{|c|}{ Phases } \\
\hline & & & $\mathbf{I}$ & II & III & IV \\
\hline $\mathbf{T 1}$ & $\mathrm{ZnSo}_{4}$ & 30 & $87.68 \pm 2.89$ & $87.05 \pm 1.60^{\mathrm{a}}$ & $87.32 \pm 1.77^{\mathrm{a}}$ & $84.68 \pm 0.63^{a}$ \\
\hline $\mathbf{T 2}$ & $\mathrm{ZnSo}_{4}$ & 60 & $89.24 \pm 2.64$ & $88.21 \pm 0.95^{\mathrm{a}}$ & $88.30 \pm 0.85^{\mathrm{ab}}$ & $87.51 \pm 1.11^{\mathrm{ab}}$ \\
\hline T3 & Zn-Met & 30 & $90.29 \pm 1.88$ & $90.34 \pm 1.13^{\mathrm{ab}}$ & $89.58 \pm 1.93^{a b c}$ & $88.94 \pm 1.61^{b c}$ \\
\hline T4 & Zn-Met & 60 & $91.34 \pm 1.83$ & $92.38 \pm 0.94^{b c}$ & $91.16 \pm 1.88^{a b c}$ & $90.38 \pm 1.09^{b c}$ \\
\hline T5 & Nano Zinc & 15 & $91.13 \pm 1.31$ & $93.20 \pm 1.50^{b c}$ & $91.53 \pm 0.72^{a b c}$ & $90.68 \pm 1.27^{b c}$ \\
\hline T6 & Nano Zinc & 30 & $92.25 \pm 1.59$ & $93.67 \pm 0.94^{b c}$ & $92.32 \pm 0.47^{b c}$ & $91.21 \pm 0.58^{\mathrm{cd}}$ \\
\hline T7 & Nano Zinc & 60 & $94.25 \pm 1.21$ & $94.21 \pm 0.85^{c}$ & $93.46 \pm 0.73^{c}$ & $93.97 \pm 0.78^{d}$ \\
\hline
\end{tabular}

Means having same superscript do not differ significantly 
Table.4 Effect of supplementing $\mathrm{ZnSo}_{4}$, Zn-Met and nano zinc on hatchability percentage of White Plymouth Rock bird

\begin{tabular}{|c|c|c|c|c|c|c|}
\hline \multirow{3}{*}{$\begin{array}{l}\text { Treat- } \\
\text { ment }\end{array}$} & \multirow{3}{*}{$\begin{array}{c}\text { Zinc } \\
\text { source }\end{array}$} & \multirow{3}{*}{$\begin{array}{l}\text { Level } \\
\text { (mg/kg) }\end{array}$} & \multicolumn{4}{|c|}{ Total Egg Set (TES)/ Fertile Egg Set (FES) } \\
\hline & & & \multicolumn{4}{|c|}{ Phases } \\
\hline & & & I & II & III & IV \\
\hline $\mathbf{T 1}$ & $\mathrm{ZnSo}_{4}$ & 30 & $\begin{array}{l}74.19 \pm 3.94^{\mathrm{a}} \\
84.42 \pm 2.31^{\mathrm{a}}\end{array}$ & $\begin{array}{l}74.29 \pm 1.57^{\mathrm{a}} \\
85.41 \pm 1.97\end{array}$ & $\begin{array}{l}74.70 \pm 1.16^{\mathrm{a}} \\
85.60 \pm 0.96^{\mathrm{a}}\end{array}$ & $\begin{array}{l}71.85 \pm 1.21^{\mathrm{a}} \\
84.86 \pm 1.57\end{array}$ \\
\hline $\mathbf{T} 2$ & $\mathrm{ZnSo}_{4}$ & 60 & $\begin{array}{c}76.01 \pm 3.16^{\mathrm{ab}} \\
85.09 \pm 1.68^{\mathrm{a}}\end{array}$ & $\begin{array}{c}75.44 \pm 0.86^{a b} \\
85.53 \pm 0.52\end{array}$ & $\begin{array}{c}76.10 \pm 0.73^{\mathrm{ab}} \\
86.18 \pm 0.42^{\mathrm{a}}\end{array}$ & $\begin{array}{c}75.02 \pm 2.44^{\mathrm{ab}} \\
85.71 \pm 2.50\end{array}$ \\
\hline T3 & $\begin{array}{l}\text { Zn- } \\
\text { Met }\end{array}$ & 30 & $\begin{array}{l}77.52 \pm 2.02^{\mathrm{abc}} \\
85.84 \pm 1.11^{\mathrm{ab}}\end{array}$ & $\begin{array}{c}78.66 \pm 0.89^{b c} \\
87.15 \pm 1.88\end{array}$ & $\begin{array}{l}77.44 \pm 0.96^{\mathrm{ab}} \\
86.51 \pm 0.89^{\mathrm{a}}\end{array}$ & $\begin{array}{c}76.00 \pm 0.92^{b c} \\
85.52 \pm 1.32\end{array}$ \\
\hline T4 & $\begin{array}{l}\text { Zn- } \\
\text { Met }\end{array}$ & 60 & $\begin{array}{l}79.98 \pm 1.45^{\mathrm{abc}} \\
87.58 \pm 0.62^{\mathrm{abc}}\end{array}$ & $\begin{array}{c}80.91 \pm 1.91^{\mathrm{cd}} \\
87.55 \pm 1.48\end{array}$ & $\begin{array}{l}79.39 \pm 1.87^{\mathrm{bc}} \\
87.06 \pm 0.55^{\mathrm{ab}}\end{array}$ & $\begin{array}{c}78.58 \pm 1.01^{b c} \\
86.97 \pm 1.20\end{array}$ \\
\hline T5 & $\begin{array}{l}\text { Nano } \\
\text { Zinc }\end{array}$ & 15 & $\begin{array}{l}80.39 \pm 0.96^{\mathrm{abc}} \\
88.24 \pm 0.98^{\mathrm{abc}}\end{array}$ & $\begin{array}{c}80.84 \pm 1.37^{\mathrm{cd}} \\
86.73 \pm 0.82\end{array}$ & $\begin{array}{l}79.92 \pm 1.37^{\mathrm{bc}} \\
87.29 \pm 1.13^{\mathrm{ab}}\end{array}$ & $\begin{array}{c}78.36 \pm 1.17^{b c} \\
86.52 \pm 2.20\end{array}$ \\
\hline T6 & $\begin{array}{l}\text { Nano } \\
\text { Zinc }\end{array}$ & 30 & $\begin{array}{l}83.03 \pm 2.47^{b c} \\
89.94 \pm 1.32^{b c}\end{array}$ & $\begin{array}{c}82.64 \pm 1.64^{\mathrm{cd}} \\
88.22 \pm 1.59\end{array}$ & $\begin{array}{l}81.77 \pm 1.75^{\mathrm{cd}} \\
88.55 \pm 1.59^{\mathrm{ab}}\end{array}$ & $\begin{array}{l}80.12 \pm 1.21^{c} \\
87.85 \pm 1.42\end{array}$ \\
\hline T7 & $\begin{array}{l}\text { Nano } \\
\text { Zinc }\end{array}$ & 60 & $\begin{array}{l}85.05 \pm 0.98^{c} \\
90.26 \pm 0.82^{c}\end{array}$ & $\begin{array}{l}84.70 \pm 1.16^{\mathrm{d}} \\
89.94 \pm 1.66\end{array}$ & $\begin{array}{l}84.19 \pm 1.18^{d} \\
90.01 \pm 0.93^{b}\end{array}$ & $\begin{array}{l}84.78 \pm 0.94^{d} \\
90.24 \pm 1.15\end{array}$ \\
\hline
\end{tabular}

Means having same superscript do not differ significantly

Table.5 Effect of supplementing $\mathrm{ZnSo}_{4}, \mathrm{Zn}-\mathrm{Met}$ and nano zinc on external egg quality parameters of White Plymouth Rock bird

\begin{tabular}{|c|c|c|c|c|c|c|}
\hline \multirow[t]{3}{*}{ Treatment } & \multirow{3}{*}{$\begin{array}{c}\text { Zinc } \\
\text { sourc } \\
\text { e }\end{array}$} & \multirow{3}{*}{$\begin{array}{c}\text { Level } \\
(\mathrm{mg} / \mathrm{kg})\end{array}$} & \multicolumn{4}{|c|}{ Egg weight (g)/shape index } \\
\hline & & & \multicolumn{4}{|c|}{ Weeks } \\
\hline & & & $37^{\text {th }}$ & $42^{\text {nd }}$ & $47^{\text {th }}$ & $52^{\text {nd }}$ \\
\hline \multirow[t]{2}{*}{ T1 } & $\mathrm{ZnSo}_{4}$ & 30 & $59.62 \pm 0.47^{\mathrm{a}}$ & $58.98 \pm 0.69^{\mathrm{a}}$ & $58.07 \pm 0.64^{\mathrm{a}}$ & $57.10 \pm 0.87^{\mathrm{a}}$ \\
\hline & & & $75.09 \pm 0.61^{\mathrm{a}}$ & $74.87 \pm 0.45^{\mathrm{a}}$ & $73.83 \pm 0.65^{\mathrm{a}}$ & $72.74 \pm 0.90^{\mathrm{a}}$ \\
\hline \multirow[t]{2}{*}{$\mathbf{T} 2$} & $\mathrm{ZnSo}_{4}$ & 60 & $61.52 \pm 0.88^{\mathrm{ab}}$ & $60.84 \pm 0.58^{\mathrm{ab}}$ & $59.85 \pm 0.54^{b}$ & $58.68 \pm 0.54^{\mathrm{ab}}$ \\
\hline & & & $77.92 \pm 1.72^{\mathrm{ab}}$ & $75.98 \pm 0.90^{\mathrm{ab}}$ & $74.94 \pm 0.85^{\mathrm{ab}}$ & $73.78 \pm 0.86^{\mathrm{ab}}$ \\
\hline \multirow[t]{2}{*}{ T3 } & Zn- & 30 & $62.98 \pm 0.72^{b c}$ & $61.42 \pm 0.85^{b}$ & $60.66 \pm 0.64^{b c}$ & $59.74 \pm 0.66^{\mathrm{bc}}$ \\
\hline & Met & & $78.00 \pm 1.14^{\mathrm{ab}}$ & $76.04 \pm 0.48^{\mathrm{ab}}$ & $75.16 \pm 0.97^{\mathrm{ab}}$ & $74.81 \pm 0.90^{\mathrm{ab}}$ \\
\hline \multirow[t]{2}{*}{ T4 } & Zn- & 60 & $63.12 \pm 0.91^{b c}$ & $62.24 \pm 0.95^{\mathrm{bc}}$ & $61.74 \pm 0.73^{\mathrm{cd}}$ & $61.62 \pm 0.69^{\mathrm{cd}}$ \\
\hline & Met & & $79.08 \pm 1.38^{\mathrm{b}}$ & $77.37 \pm 0.90^{\mathrm{b}}$ & $76.28 \pm 0.57^{\mathrm{bc}}$ & $75.35 \pm 0.65^{b c}$ \\
\hline \multirow[t]{2}{*}{ T5 } & Nano & 15 & $63.54 \pm 0.61^{b c}$ & $62.93 \pm 0.68^{\mathrm{bc}}$ & $61.76 \pm 0.64^{\mathrm{cd}}$ & $61.52 \pm 0.63^{\mathrm{cd}}$ \\
\hline & Zinc & & $79.32 \pm 1.51^{\mathrm{b}}$ & $77.31 \pm 0.94^{b}$ & $77.57 \pm 0.93^{\mathrm{cd}}$ & $75.76 \pm 0.94^{b c}$ \\
\hline \multirow[t]{2}{*}{ T6 } & Nano & 30 & $64.05 \pm 1.32^{\mathrm{bc}}$ & $64.14 \pm 0.50^{c}$ & $62.87 \pm 0.62^{\mathrm{de}}$ & $62.27 \pm 0.76^{\mathrm{de}}$ \\
\hline & Zinc & & $81.27 \pm 0.66^{\mathrm{bc}}$ & $80.85 \pm 0.87^{\mathrm{c}}$ & $79.23 \pm 0.72^{\mathrm{d}}$ & $77.68 \pm 0.65^{\mathrm{cd}}$ \\
\hline \multirow[t]{2}{*}{ T7 } & Nano & 60 & $65.26 \pm 0.36^{c}$ & $66.25 \pm 0.64^{d}$ & $64.35 \pm 0.43^{\mathrm{e}}$ & $64.15 \pm 0.61^{\mathrm{e}}$ \\
\hline & Zinc & & $83.04 \pm 0.89^{c}$ & $82.60 \pm 0.92^{c}$ & $81.56 \pm 0.41^{\mathrm{e}}$ & $79.35 \pm 0.57^{d}$ \\
\hline
\end{tabular}

Means having same superscript do not differ significantly 
Table.6 Effect of supplementing $\mathrm{ZnSo}_{4}$, Zn-Met and nano zinc on yolk and albumen index of White Plymouth Rock bird

\begin{tabular}{|c|c|c|c|c|c|c|}
\hline \multirow{3}{*}{$\begin{array}{l}\text { Treat- } \\
\text { ment }\end{array}$} & \multirow{3}{*}{$\begin{array}{c}\text { Zinc } \\
\text { source }\end{array}$} & \multirow{3}{*}{$\begin{array}{c}\text { Level } \\
\text { (mg/kg) }\end{array}$} & \multicolumn{4}{|c|}{ Yolk index/albumen index } \\
\hline & & & \multicolumn{4}{|c|}{ Weeks } \\
\hline & & & $37^{\text {th }}$ & $42^{\text {nd }}$ & $47^{\text {th }}$ & $52^{\text {nd }}$ \\
\hline T1 & $\mathrm{ZnSo}_{4}$ & 30 & $\begin{array}{l}0.35 \pm 0.009^{\mathrm{a}} \\
0.06 \pm 0.001^{\mathrm{a}}\end{array}$ & $\begin{array}{l}0.351 \pm 0.004^{\mathrm{a}} \\
0.060 \pm 0.002^{\mathrm{a}}\end{array}$ & $\begin{array}{l}0.348 \pm 0.005^{\mathrm{a}} \\
0.059 \pm 0.001^{\mathrm{a}}\end{array}$ & $\begin{array}{l}0.340 \pm 0.006^{\mathrm{a}} \\
0.057 \pm 0.002^{\mathrm{a}}\end{array}$ \\
\hline $\mathbf{T 2}$ & $\mathrm{ZnSo}_{4}$ & 60 & $\begin{array}{c}0.36 \pm 0.005^{\mathrm{ab}} \\
0.06 \pm 0.001^{\mathrm{a}}\end{array}$ & $\begin{array}{l}0.361 \pm 0.005^{\mathrm{ab}} \\
0.061 \pm 0.001^{\mathrm{ab}}\end{array}$ & $\begin{array}{l}0.355 \pm 0.004^{\mathrm{ab}} \\
0.061 \pm 0.001^{\mathrm{ab}}\end{array}$ & $\begin{array}{c}0.350 \pm 0.004^{\mathrm{ab}} \\
0.058 \pm 0.009^{\mathrm{a}}\end{array}$ \\
\hline T3 & $\begin{array}{l}\text { Zn- } \\
\text { Met }\end{array}$ & 30 & $\begin{array}{c}0.37 \pm 0.008^{\mathrm{abc}} \\
0.06 \pm 0.001^{\mathrm{a}}\end{array}$ & $\begin{array}{c}0.370 \pm 0.006^{\mathrm{ab}} \\
0.063 \pm 0.001^{\mathrm{abc}}\end{array}$ & $\begin{array}{l}0.361 \pm 0.007^{\mathrm{abc}} \\
0.060 \pm 0.001^{\mathrm{abc}}\end{array}$ & $\begin{array}{c}0.366 \pm 0.008^{\mathrm{abc}} \\
0.059 \pm 0.001^{\mathrm{ab}}\end{array}$ \\
\hline T4 & $\begin{array}{l}\text { Zn- } \\
\text { Met }\end{array}$ & 60 & $\begin{array}{c}0.39 \pm 0.008^{\mathrm{bcd}} \\
0.06 \pm 0.001^{\mathrm{ab}}\end{array}$ & $\begin{array}{c}0.381 \pm 0.007^{\mathrm{bc}} \\
0.064 \pm 0.001^{\mathrm{abc}}\end{array}$ & $\begin{array}{c}0.372 \pm 0.007^{\mathrm{bc}} \\
0.06 \pm 0.001^{\mathrm{abc}}\end{array}$ & $\begin{array}{c}0.374 \pm 0.007^{\mathrm{bcc}} \\
0.061 \pm 0.002^{\mathrm{ab}}\end{array}$ \\
\hline T5 & $\begin{array}{l}\text { Nano } \\
\text { Zinc }\end{array}$ & 15 & $\begin{array}{l}0.39 \pm 0.01^{\mathrm{bcd}} \\
0.06 \pm 0.002^{\mathrm{ab}}\end{array}$ & $\begin{array}{c}0.38 \pm 0.011^{\mathrm{bc}} \\
0.064 \pm 0.002^{\mathrm{abc}}\end{array}$ & $\begin{array}{c}0.373 \pm 0.011^{b c} \\
0.063 \pm 0.002^{a b c}\end{array}$ & $\begin{array}{c}0.37 \pm 0.01^{\mathrm{bcd}} \\
0.061 \pm 0.001^{\mathrm{ab}}\end{array}$ \\
\hline T6 & $\begin{array}{l}\text { Nano } \\
\text { Zinc }\end{array}$ & 30 & $\begin{array}{c}0.40 \pm 0.06^{\mathrm{cd}} \\
0.07 \pm 0.001^{\mathrm{bc}}\end{array}$ & $\begin{array}{l}0.392 \pm 0.006^{\mathrm{cd}} \\
0.066 \pm 0.001^{\mathrm{bc}}\end{array}$ & $\begin{array}{l}0.383 \pm 0.006^{\mathrm{cd}} \\
0.064 \pm 0.001^{\mathrm{bc}}\end{array}$ & $\begin{array}{l}0.381 \pm 0.011^{\mathrm{cd}} \\
0.063 \pm 0.001^{\mathrm{bc}}\end{array}$ \\
\hline T7 & $\begin{array}{l}\text { Nano } \\
\text { Zinc }\end{array}$ & 60 & $\begin{array}{c}0.42 \pm 0.01^{d} \\
0.073 \pm 0.001^{c}\end{array}$ & $\begin{array}{c}0.410 \pm 0.007^{d} \\
0.067 \pm 0.04^{c}\end{array}$ & $\begin{array}{l}0.398 \pm 0.008^{d} \\
0.067 \pm 0.001^{c}\end{array}$ & $\begin{array}{c}0.39 \pm 0.01^{\mathrm{d}} \\
0.066 \pm 0.001^{\mathrm{c}}\end{array}$ \\
\hline
\end{tabular}

Means having same superscript do not differ significantly

Hatchability for small eggs is lower than medium and large eggs, which is following the present results of the nano zinc supplemented group which was having a higher egg weight as compared to other groups. The hatchability on TES was recorded highest in $\mathrm{T}_{7}$ (Nano zinc, $60 \mathrm{mg} / \mathrm{kg}$ ) which differed significantly when compared to other treatment groups.

The lowest hatchability on the TES percentage of 72.85 was recorded for the $T_{1}$ group. The hatchability on TES percentages of $\mathrm{Zn}$-Met treatment groups was comparable to that of nano zinc fed groups $\left(\mathrm{T}_{5}\right.$ and $\left.\mathrm{T}_{6}\right)$ groups. Among $\mathrm{ZnSo}_{4}$ groups at different levels were comparable for hatchability on TES percentage. This conclusion regarding the improvement of hatchability is supported by the reports Hassan et al., (2003), wherein an increase in hatchability percentage was recorded from organic trace minerals supplementation.
The Hatchability on FES in the first phase of the study was significantly $(\mathrm{P} \leq 0.05)$ highest 90.26 in the $\mathrm{T}_{7}$ group when compared to other treatment groups. But, the difference was comparable with nano zinc supplementation at the levels of 15 and $30 \mathrm{mg} / \mathrm{kg}$ and also with the $\mathrm{Zn}$-Met treatment group at the level of 60 $\mathrm{mg} / \mathrm{kg}$. Among $\mathrm{ZnSo}_{4}$ groups, the difference was comparable between them with the lowest value recorded for the $\mathrm{T}_{1}$ group.

The higher percentage of hatchability on FES in the case of $\mathrm{T}_{7}$ can substantiate by a higher egg weight in the respective treatment group. (Abiola et al., 2008; Malago and Baitilwake, 2009) showed that there was a close correlation between egg size and chick hatching weight. Breeder factors that affect hatchability include nutrition, health, egg size, egg weight, and quality King'ori (2011). The results are contradictory to the findings of Hudson et al., (2004) reported a significant reduction in early embryonic mortality when breeder hens were fed a Zn-amino acid 
complex as the only source of $\mathrm{Zn}$ supplementation in the diet.

During phase II-IV, the value for hatchability on FES was statistically insignificant $(\mathrm{P} \leq 0.05)$ in all the treatment groups. The findings of the present study are in line with King'ori (2011) reported that supplementation of zinc had little or no effect on the hatchability. Age is one of the factors which could affect the percent hatchability. Hatchability for eggs of older breeders decreases because of a change in egg quality and failure to adjust the incubation condition. It was further stated that as the hen ages, the albumen quality, which is the main source protein to the embryo development deteriorates Slamony et al., (2015).

\section{External egg quality parameters}

In the eggs collected during different weeks of egg production, the egg weight was recorded significantly higher in $\mathrm{T}_{7}$ (Nano zinc, $60 \mathrm{mg} / \mathrm{kg}$ ) with the lowest egg weight in $\mathrm{T}_{1}\left(\mathrm{ZnSo}_{4}, 30 \mathrm{mg} / \mathrm{kg}\right)$ between different treatment groups depicted (Table 5). The differences among the $\mathrm{ZnSo}_{4}$ groups were insignificant $(\mathrm{P} \leq 0.05)$ with a higher weight in $\mathrm{T}_{2}\left(\mathrm{ZnSo}_{4}, 60 \mathrm{mg} / \mathrm{kg}\right)$. The differences in egg weights of Zn-Met treated groups were comparable to that of nano zinc supplemented groups. The egg weight in $\mathrm{Zn}-\mathrm{Met}$ supplemented at the level of $60 \mathrm{mg} / \mathrm{kg}\left(\mathrm{T}_{4}\right)$ was comparable to that of nano zinc supplemented groups at the level of 15 and 30 $\mathrm{mg} / \mathrm{kg}$. Similar findings were also reported by (Slamony et al., 2015; Durmus et al., 2004), wherein dietary supplementation with zinc resulted in a significant increase in egg weight and the concentration of zinc in eggs. The finding is also supported by the results of (Amem and Daraji 2011) reported an increase in egg weight by increasing the $\mathrm{Zn}$ concentration in the diet. The better changes observed with nano zinc treated groups at the level of $60 \mathrm{mg} / \mathrm{kg}$ in egg weight of this study may be attributed to that both of nano zinc improved nutrient digestibility and metabolism. While Kidd et al., (1992) found that there was no significant effect on egg weight upon addition of zinc to the diet of broiler parent stock.

A significantly $(\mathrm{P} \leq 0.05)$ higher shape index value was recorded for $\mathrm{T}_{7}$ (Nano zinc, 60 $\mathrm{mg} / \mathrm{kg}$ ) in comparison to other treatment groups with the lowest value of shape index in $T_{1}$ group which was supplemented with $\mathrm{ZnSo}_{4}$ at the level of $30 \mathrm{mg} / \mathrm{kg}$ in all the eggs collected during different weeks of the study period. The shape index values for $T_{5}$ and $T_{6}$ were comparable with a higher value at the level of $30 \mathrm{mg} / \mathrm{kg}$ nano zinc in the diet. Among $\mathrm{ZnSO}_{4}$ treatment groups, the shape index values were comparable at all the weeks of the study period. In general, a long and narrow egg of any size would have a low index and a short and broad egg (whether large or small) would have a high index. The higher shape index value of indigenous chicken might be due to the broad and short size and shape of the eggs Pathak et al., (2016). In the present study eggs of $T_{7}$ were found to be broader with a relatively shorter length contributing to a higher shape index. The result of the present study is in contrast to the reports of (Slamony et al., 2015; Bahakaim et al., 2014) exhibited that no significant differences in egg shape index with the dietary organic zinc supplementation of Golden Montazah hens.

\section{Internal egg quality}

The yolk index was highest (0.422) in $\mathrm{T}_{7}$ and lowest (0.35) in the $T_{1}$ group supplemented with $\mathrm{ZnSo}_{4}$ at the level of $30 \mathrm{mg} / \mathrm{kg}$ (Table 6). The difference was comparable among the Zn-Met treatment groups at the levels of 30 and $60 \mathrm{mg} / \mathrm{kg}$. The yolk index values of $\mathrm{ZnSo}_{4}$ treatment groups were comparable to 
that of the Zn-Met group at the level of 30 $\mathrm{mg} / \mathrm{kg}$. Among $\mathrm{ZnSo}_{4}$ treatment groups, the values were comparable. The reports of the present study are in line with the reports of (Lim and Paik 2003; Kout El-Koub et al., 2004) reported zinc methionine chelate supplementation, did not affect internal egg quality. (Slamony et al., 2015) and (Bahakaim et al., 2014) also exhibited no significant differences in the yolk index with the dietary organic zinc supplementation of Golden Montazah hens.

During the $37^{\text {th }}$ week, the albumen index ranged from (0.061) in $T_{1}$ and (0.073) in $T_{7}$ groups. The height of the albumen was increased significantly in the eggs of the $T_{7}$ treatment group. Albumen index was significantly $(\mathrm{P} \leq 0.05)$ different in the $\mathrm{T}_{7}$ group when compared to other groups except in $\mathrm{T}_{6}$. Among the $\mathrm{ZnSo}_{4}$ supplemented groups at a different level and Zn-Met groups the differences in albumen indices were comparable. The albumen index value of $\mathrm{T}_{4}$ (Zn-Met, $60 \mathrm{mg} / \mathrm{kg}$ ) was comparable to that of nano zinc supplemented groups in $T_{5}$ and $\mathrm{T}_{6}$. The present findings are corroborated with the reports of (Slamony et al., 2015; Bahakaim et al., 2014) exhibited that no significant differences in the albumen index with the dietary organic zinc supplementation of Golden Montazah hens. The findings are in contrast to the reports of (Tabatabaie et al., 2007) reported an increase of albumen height in the eggs of the hens supplemented with organic zinc. The reason for the increase in the albumen index might be attributed to increased albumen height in the $\mathrm{T}_{7}$ treatment. Another reason might be attributed as Zinc plays a role in the magnum during the deposition of albumen and in the isthmus where eggshell membranes are produced. Findings for the increase in height of albumen in the treatment group compared to the control group were also reported by (Tabatabaie et al., 2007) who supplemented the diet with organic zinc at a different level.

A significantly $(\mathrm{P} \leq 0.05)$ higher eggshell thickness (0.42) was recorded for $\mathrm{T}_{7}$ in comparison to other treatment groups. The eggshell thickness was found to be lowest (0.36) in the $T_{1}$ group. The value of eggshell thickness in $T_{1}$ and $T_{2}$ were comparable. A comparable value of eggshell thickness was observed between $\mathrm{Zn}$-Met treated groups and Nano zinc supplemented groups at the level of 15 and $30 \mathrm{mg} / \mathrm{kg}$. Among $\mathrm{ZnSo}_{4}$ treatment groups, the values were comparable to each other. The findings of the present study are correlated with the reports of Bahakaim et al., (2014) exhibited shell thickness was significantly improved as a result of using organic $\mathrm{Zn}$ in the diet of Golden Montazah birds. The findings of Klecker et al., (2002) also supported present findings, who reported that the substitution of $20-40 \%$ supplemental inorganic $\mathrm{Zn}$ with their chelates increased eggshell thickness.

Among different sources used in the study, nano zinc particles are the source in which bioavailability is highest, due to their extremely small size and unique physical properties Sahoo et al., (2014). The results for increase shell thickness might be attributed to the importance of zinc function in the formation of the egg. Zinc supplementation also has been reported to improve eggshell quality because it is a component of the carbonic anhydrase enzyme, which supplies the carbonate ions during eggshell formation Innocenti et al., (2004).

The Haugh unit value ranged from 72.41 in $\mathrm{T}_{1}$ to 78.44 in $\mathrm{T}_{7}$. The difference was recorded significant $(\mathrm{P} \leq 0.05)$ in $\mathrm{T}_{7}$ when compared to other treatment groups except with $\mathrm{T}_{6}$. Among Zn-Met groups, the differences were statistically insignificant and were comparable to that of $\mathrm{T}_{5}$ and $\mathrm{T}_{6}$. The increase 
in the Haugh unit in the present study is in agreement with the report of Sahin and (Kucuk 2003; Idowu et al., 2011) who reported that zinc supplementation positively affected the Haugh unit. Concerning the interaction between $\mathrm{Zn}$ levels and sources, it was observed that the Haugh unit was significantly affected. Supplementation of nano zinc at $60 \mathrm{mg} / \mathrm{kg} \mathrm{Zn}$ gave the preferable value in the Haugh unit. The reason might be attributed to an increase in albumen height in the $\mathrm{T}_{7}$ group.

In conclusion the Hen House and Hen Day Egg production were increased with nano zinc supplementation at a higher level of @60mg/kg. The fertility, hatchability on Total Egg set, and Fertile Egg Set were improved on an increasing level of zinc supplementation in all the sources of zinc; however, a better percentage of the aforementioned parameters were with nano zinc supplementation at a higher level. The egg quality traits were also improved with a higher level of nano zinc supplementation.

\section{Acknowledgement}

The corresponding author is thankful to Department of Science and Technology, Government of India for providing the funds under INSPIRE fellowship for $\mathrm{PhD}$ degree program. The authors acknowledged M/S Zeus Biotech Private Limited, Mysore for organic zinc (Zn-methionine chelate- 8\%), M/S Pristine organics Pvt. Ltd, Bengaluru for supplying inorganic source (Zinc Sulphate26\%) and Ritus Nutraceuticals, Chennai for the supply of Nano zinc.

\section{Authors' contributions}

Siddhartha Shankar Pathak and Biju Borah were involved in conduct of the trial while Prasoon Sagunan helped in preparation of manuscript.

\section{References}

AOAC. 2012. Official Methods of Analysis of AOAC International 19th Edn. Vol. I., Mineral salts in animal feeds: Preparation of sample solutions. Edt. Dr. George. W. Latimer Jr. pp: 60-61.

Abiola, S.S. 1999. Effects of turning frequency of hen's egg in electric tabletype incubator on weight losses, hatchability and mortality. Nig. Agr. J. 30: 77-82.

Amen, M. H. M. and Al-Daraji, H. J. 2011. Zinc improves egg quality in cobb500 broiler breeder females. Int. J. Poult. Sci.10(6): 471-476.

Bahakaim, A. S. A., Abdel, H. A., Magied, S. M. H., Osman, Omar, A. S. Abdelmalak, N. Y. and Nehad, A.R. 2014. Effect of using different levels and sources of zinc in layer's diets on egg zinc enrichment. Egypt. Poult. Sci. 34(i): 39-56.

Bedwal, R. S. and Bahugana, A. 1994. Zinc, copper and selenium in reproduction. Experientaia. 50: 626-640.

Bureau of Indian Standards, 2007. Nutrient requirement of poultry. Bureau of Indian Standards, I.S. 13574:1992.

Duncan, D. B. 1995. Multiple range and multiple f-tests. Biometrics 11: 1- 42.

Durmus I, C., Atasoglu, C., Ertas, M. S. and Kaya, M. 2004. Effect of increasing zinc concentration in the diets of brown parent stock layers on various production and hatchability rates. Arch tierz dummerstorf. 5(47): 483-489.

Fakler, T. M., Ward, T. L. and Kuhl, H. J. 2002. Zinc amino acids complexes (avila zn) improve layer production and egg quality. Poult. Sci.: 81-85.

Feng, M., Wanf, Z. S, Zhou, A. G., and Ai, D.W. 2009. Effects of different sizes of nanometer zinc oxide on the proliferation and cell integrity of mice duodenum-epithelial cells in primary 
culture. Pak. J. Nutri. 8: 1164-1166.

Guo, Y. M., Yang, R., Yuan, J., Ward, T. L. and Fakler, T. M. 2002. Effect of Availar-Zn and $\mathrm{Znso}_{4}$ on laying hen performance and egg quality. Poult. Sci. 81 (suppl. 1): 40. (Abstr.).

Hassan, R. A., Ganzoury, E. H., Abd El Ghany, El F. A. and Shehata. M. A. 2003. Influence of dietary zinc supplementation with methionine or microbial phytase enzyme on productive and reproductive performance for mandarah strain. Egypt. Poult. Sci. 23: 761-785.

Hudson, B. P., Dozier, W. A., Wilson, J. L., Sander, J. E. and Ward., T. L. 2004. Reproductive performance and immune status of caged broiler breeder hens provided diets supplemented with either inorganic or organic sources of zinc from hatching to 65 week of age. $J$. Appl. Poult. Res. 13: 349-359.

Idowu, O. M. O., Ajuwon, R. O., Oso, A. O. and Akinloye, O. A. 2011. Effects of zinc supplementation on laying performance, serum chemistry and $\mathrm{Zn}$ residue in tibia bone, liver, excreta and egg shell of laying hens. Int. J. Poult. Sci. 10 (3): 225-230.

Innocenti, A., Zimmerman, S., Ferry, J. G., Scozzafava, A. and Supuran, C. T. 2004. Carbonic anhydrase inhibitors. Inhibition of the zinc and cobalt gamma class enzyme from the archaeno methanosarcina thermophila with anions. Bioor. Med. Chem. Lett. 14: 3327-3331.

Kidd, M.T., N.B. Anthony and S.R. Lee. 1992. Progeny performance when dams and chicks are fed supplemental zinc. Poult. Sci. 71: 1201-1206 (abstr.)

King'ori, A.M. 2011. Review of the factors that influence egg fertility and hatchability in poultry. Int. J. Poult. Sci. 10: 483-492.

Klecker, D., Zeman, L., Jelinek, P. and
Bunesova, A. 2002. Effect of manganese and zinc chelates on the quality of eggs. Acta Univ. Agri. et Silvic. Mendelianae Brun. 50: 59-68.

Kout El-Kloub M. E., R. A. Hassan, E. H. ElGanzory and E. A. El-Abd. 2004. Effect of different sources and levels of zinc on the performance of local laying hens. Egypt. Poult. Sci. 24: 369-385.

Lim, H. S. and Paik, I. K. 2003. Effect of supplementary mineral methionine chelates $(\mathrm{Zn}, \mathrm{Cu}, \mathrm{Mn})$ on the performance and eggshell quality of laying hens. Asian-australas. J. Anim. Sci. 16(12): 1804-1808.

Malago, J. J. and Baitilwake, M. A. 2009. Egg traits, fertility, hatchability and chick survivability of Rhode Island Red, local and crossbred chickens. Tanzania Vet. J. 26: 24-36.

Mcdowell, L. R. 1992. Newly discovered and other trace elements. In: minerals in animal and human nutrition. Academic press. Inc, london. pp. 366-379.

Nys, Y., Bain, $M$ and Immerseel, F. Van. 2011. Hen nutrition for sustained egg quality. Improving the safety and quality of eggs and egg products. Volume 1: Egg chemistry, production and consumption, pp. 261-299.

Pathak, S. S., Barua, N. and Kalita, N. 2016. Comparison of egg quality characteristics of indigenous and broiler parent line crossed with indigenous chicken. Indian J. Vet. Sci. and Biotech. 11(3): 12-16.

Pathak, S., Saikia, A., and Tamuli, U. 2020. Influence of graded levels of different sources of zinc on growth performances and production economics in chicken. Int. J. Livest. Res. 10(8): 123-129.

Renema, R.A., Robinson, F. E., Proudman, J., Newcombe, A. M. and Mckay, R. I. 1999. Effects of body weight and feed allocation during sexual maturation in broiler breeder hens. Ovarian 
morphology and plasma hormone profiles. Poult. Sci.78: 629-639.

Sahin, K. and Kucuk, O. 200. Heat stress and dietary vitamin supplementation of poultry diets. Nutr. Abstr. Rev. Ser. B, Livest. Feeds and feeding 73(7): 41r$50 \mathrm{r}$.

Sahoo, A., Swain, R. K. and Mishra, S. K. 2014. Effect of inorganic, organic and nano zinc supplemented diets on bioavailability and immunity status of broilers. Int. J. Adv. Res. 2(11): 828837.

Slamony, A. E., Abdalla, E. A., Faddle, A. A., Mohamed, H. S. and Sabry, M. M. 2015. Effect of supplementing selenium and zinc in diet on some sex hormones, productive, reproductive and physiological parameters of laying golden montazah chicken during summer season. Egypt. Poult. Sci. 35(iii): 647-672.

Tabatabaie, M. M., Aliarabi, H., Saki, A. A., Ahmadi, A. and Siyar, H. S. A. 2007. Effect of different sources and levels of zinc on egg quality and laying hen performance. Pak. J. Biol. Sci. 10(19): 3476-3478.

Yang, X., Zhong, L., An, X., Zhang, N., Zhang, L., Han, J., Yao, J., Cote, C. and Sun, Y. 2012. Effects of diets supplemented with zinc and manganese on performance and related parameters in laying hens. Anim. Sci. J. 83(6): 47481.

\section{How to cite this article:}

Siddhartha Shankar Pathak, Prasoon Sagunan, Biju Borah and Prasanta Chabudhara. 2021. Evaluating the Effect of Different Sources of Zinc on Egg Production, Egg Quality, and Hatchability Traits in Chickens. Int.J.Curr.Microbiol.App.Sci. 10(02): 613-624. doi: https://doi.org/10.20546/ijcmas.2021.1002.073 